\title{
A New Adjuvant MTOM Mediates Mycobacterium tuberculosis Subunit Vaccine to Enhance Th1-Type T Cell Immune Responses and IL-2 ${ }^{+} \mathbf{T}$ Cells
}

OPEN ACCESS

Edited by:

Jeffrey K. Actor,

UTHealth Science Center,

United States

Reviewed by:

Juraj Ivanyi,

King's College London, UK Angelo Izzo,

Colorado State University, United States

*Correspondence: Xionglin Fan

xlfan@hust.edu.cn

Specialty section:

This article was submitted to Vaccines and Molecular

Therapeutics, a section of the journal Frontiers in Immunology

Received: 07 February 2017 Accepted: 02 May 2017

Published: 18 May 2017

Citation:

Yu Q, Wang X and Fan X (2017) A New Adjuvant MTOM Mediates Mycobacterium tuberculosis Subunit Vaccine to Enhance Th1-Type T Cell Immune Responses and IL-2+ $T$ Cells.

Front. Immunol. 8:585. doi: 10.3389/fimmu.2017.00585

\section{Qi Y ${ }^{1,2}$, Xiaochun Wang ${ }^{1}$ and Xionglin Fan ${ }^{1 *}$}

'Department of Pathogen Biology, School of Basic Medicine, Tongji Medical College, Huazhong University of Science \& Technology, Wuhan, China, ${ }^{2}$ School of Basic Medicine, Guiyang Traditional Chinese Medical College, Guiyang, China

The only licensed vaccine Mycobacterium bovis Bacillus Calmette-Guérin (BCG) cannot prevent the prevalence of tuberculosis (TB), which remains a major public health problem worldwide. A more effective TB vaccine than BCG is urgently needed. Subunit vaccine is a promising strategy, and suitable adjuvants will benefit the development of effective TB subunit vaccines. MTO, consisting of monophosphoryl lipid A (MPLA), trehalose-6,6'-dibehenate (TDB), and MF59, was developed as an adjuvant of TB vaccine because of its ability to evoke the Th1-type T cell responses, while it is insufficient to induce single and multifunctional IL-2+ T cells and has a limited ability to confer protection against Mycobacterium tuberculosis infection. Heat-killed Mycobacterium vaccae (Mv), which can evoke cytotoxic CD8 ${ }^{+}$and $C D 4^{+} T$ cell responses and has adjuvanticity, was, in this study, combined with MTO to produce a new adjuvant, called MTOM. The TB fusion protein Rv3407-PhoY2-Ag85A-Rv2626c-RpfB (WH121) was mixed with MTO, $\mathrm{Mv}$, and MTOM to produce three subunit vaccines, and the protective efficacy and immune responses were compared in C57BL/6 mice. WH121/MTOM provided better protection against TB than the other two vaccines, matching the performance of BCG vaccine. MTOM showed stronger ability to increase single and multifunctional IL-2+ T cells and induce Th1-type responses than MTO or Mv. Therefore, MTOM might be a promising adjuvant that could contribute to the development of TB subunit vaccines.

Keywords: adjuvant, MTOM, Mycobacterium tuberculosis, Th1-type response, IL-2 ${ }^{+}$T cells

\section{INTRODUCTION}

Mycobacterium bovis Bacillus Calmette-Guérin (BCG), an attenuated live strain derived from $M$. bovis and the only licensed vaccine for tuberculosis (TB), has been vaccinated in neonates worldwide with high coverage since the 1970s. However, TB remains the major threat among infectious diseases. There were approximately 10 million of new registered TB cases, and deaths from TB reached 1.4 million in 2015, respectively (1). Moreover, about one-third of the world's population is estimated to be latent TB infections (LTBIs). Therefore, BCG cannot provide effective protection to control the prevalence of TB (2). A more effective TB vaccine than BCG is urgently needed.

$\mathrm{CD} 4^{+} \mathrm{Th} 1$ responses play a central role in the resistance to Mycobacterium tuberculosis ( $M$. $t$ b) infection $(3,4)$. Recent studies have suggested that $\mathrm{CD} 4^{+} \mathrm{T}$ cells are required to control TB progression and sustain multifunctional CD8 ${ }^{+} \mathrm{T}$ cells during $M$. $t b$ infection of non-human primates (5). Moreover, $\mathrm{CD} 8^{+} \mathrm{T}$ cells expressing Th1 cytokines probably contribute to the control of LTBI (6). 
Although Th17 responses are involved in immune protection against $M$. $t b$ through recruiting and activating neutrophils at an early stage of infection, over-stimulation of Th17 responses leads to an exaggerated inflammation that instead contributes to tissue damage (7). Consequently, Th1-type T cell-mediated immunity is an attractive target for the development of new TB vaccines (8). Of different strategies, almost half of vaccine candidates in clinical trials belong to the type of subunit protein vaccination, which is generally accepted as a promising immunization strategy against $M . t b$ (9). However, recombinant proteins are usually poorly immunogenic and the formulation in adjuvants is required to strengthen the magnitude of the responses to the protein antigen or to alter the type of immune responses induced (10). Alum adjuvant is widely used in human vaccines and mainly induces antibody response (11). Water-in-oil adjuvant MF59 (12) and AS03 (13) promote the generation of influenza-specific antibody. The adjuvant AS04, composed of monophosphoryl lipid A (MPLA) adsorbed on aluminum salt, is utilized in human papilloma virus and hepatitis $B$ virus vaccine preparations to increase antigen-presenting cells (APCs) and the levels of IL- 6 and TNF- $\alpha$ $(14,15)$. There are indeed other adjuvants in the pipeline that are known to induce Th1 immune response (16). CAF01, containing trehalose-6, $6^{\prime}$-dibehenate (TDB), has been found to promote Th1 and Th17 immune responses in clinical Phase II trials (17). In our previous studies, we mixed MPLA and TDB in MF59 to produce a novel adjuvant, MTO. Although MTO-adjuvanted A1D4 subunit vaccine can elicit Th1-type immune response, its ability to induce single and multifunctional IL- $2^{+} \mathrm{T}$ cells is insufficient, which thus resulted in the inferior protective efficacy against $M$. $t b$ infection to BCG (18). However, these adjuvants provide insufficient enhancement to the Thl-type $\mathrm{T}$ cell immune response and IL-2 ${ }^{+}$ $\mathrm{T}$ cells. The appropriate, licensed adjuvants are required for the development of TB subunit vaccines.

Previously, Skinner et al. found that heat-killed Mycobacterium vaccae $(\mathrm{Mv})$ had adjuvanticity and could evoke cytotoxic CD8 ${ }^{+}$ and $\mathrm{CD} 4^{+} \mathrm{T}$ cells responses in immunized mice (19). In our earlier work, mouse immunization with live Mv strains induced protective immune responses against $M$. $t b$ (20). In order to improve the adjuvant effect of MTO, we first incorporated heat-killed Mv in MTO to construct a new adjuvant, MTOM. A polyprotein Rv3407-PhoY2-Ag85A-Rv2626c-RpfB, which was based on five immunodominant antigens expressed during different stages of TB infection, WH121 for short, that had previously been shown in mice to be a promising subunit vaccine candidate (21), was mixed with the adjutants MTO, Mv, and MTOM, and the immune responses and the protective efficacy on mice exposed to $M$. $t b$ were observed and compared.

\section{RESULTS}

\section{MTOM-Adjuvanted WH121 Subunit Vaccine Affords Improved Protective Efficacy}

To compare the protection of WH121 protein combined with different adjuvants, C57BL/6 mice were challenged intranasally with live $M . t b$ strain $\mathrm{H} 37 \mathrm{Rv}$ after the last immunization.
Protective efficacy was assessed by comparison of the bacterial load in lung and spleen and by the lung pathology at 1 month post infection. As shown in Figure 1A, the highest bacterial load in both lung and spleen was observed in the PBS control group. Either adjuvant MTO, Mv, or WH121 alone also slightly inhibited the growth of $M$. $t b$ in both organs, but the effects were inferior to those offered by MTOM $(P<0.05)$. C57BL/6 mice vaccinated with WH121 subunit vaccines showed significantly lower colony-forming unit (CFU) values in the lung and spleen than mice treated with adjuvant alone. Importantly, the bacterial load in WH121/MTOM group was lower than those in WH121/ MTO and WH121/Mv groups, and similar to the results of the BCG group.

As shown in Figure 1B, mice in the PBS control group presented the most severe histopathology with extensive fibrosis, perivasculitis, pulmonary alveolitis, and lymphocyte infiltrates, and acid-fast (AF)-positive bacilli were found throughout the whole lung section. Mice in the adjuvant or WH121 protein alone groups also showed dispersed AF-positive bacilli in the lung and exhibited similar, less severe pathological changes to those observed in the PBS control mice. In contrast, the pulmonary lesions and inflammation of mice in the WH121/ MTO and WH121/Mv groups were substantially reduced and a few AF-positive bacilli were detected in the alveolar tissue. Remarkably, the mice vaccinated with WH121/MTOM exhibited less pronounced pathological manifestation than those vaccinated with WH121/MTO and WH121/Mv. They were comparable to the effects observed in BCG-immunized mice (Figure 1B). All these results demonstrated that MTOM-adjuvanted WH121 performed better than the combinations of MTO or Mv and WH121, and its protective efficacy matched with that of BCG.

\section{WH121/MTOM Subunit Vaccine Induced Stronger Antibody Responses}

To determine the levels of antibody induced by different WH121 vaccines, mice were vaccinated twice, 3 weeks apart with WH121/ MTO, WH121/Mv, or WH121/MTOM. Six weeks after the final vaccination, WH121-specific antibodies, including IgG, IgG1, and IgG2a, were titrated by ELISA.

As shown in Figure 2, there was no WH121-specific antibody response to adjuvant $\mathrm{MTO}, \mathrm{Mv}$, or $\mathrm{MTOM}$ alone. Compared to BCG, mice vaccinated with WH121/MTOM produced higher levels of WH121-specific IgG, IgG1, and IgG2a $(P<0.001)$. The ratio of IgG2a/IgG1 substantially increased (Figure 2A), which indicated that WH121/MTOM elicited a shift of the IgG subclass toward a Th1-type response. Furthermore, WH121/ MTOM induced higher levels of these antibodies than WH121/ MTO or WH121/Mv (Figure 2B), and the conversion trend of the IgG subclass toward Th1-type responses was more obvious (Figure 2B).

\section{Th1 Cytokines in Response to Subunit WH121/MTOM}

In this study, IFN- $\gamma$, TNF- $\alpha$, and IL- 2 released by Th1 cells were detected to explore the effects of different adjuvant WH121 formulations on Th1-type immune responses. The results showed the 


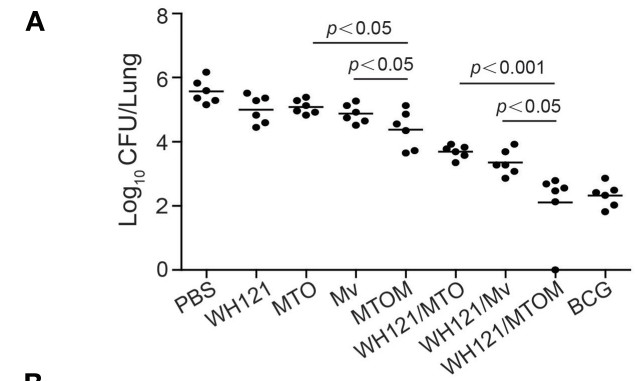

B
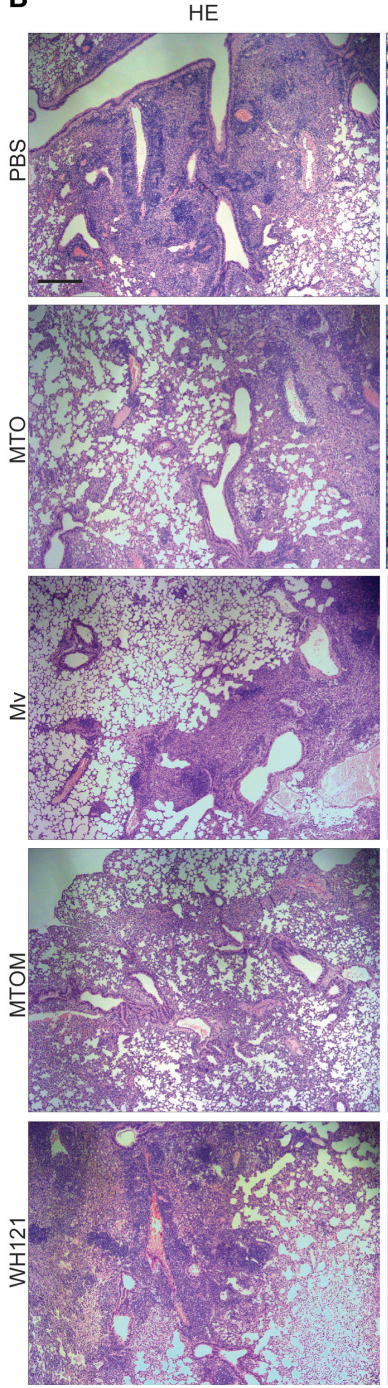

AF
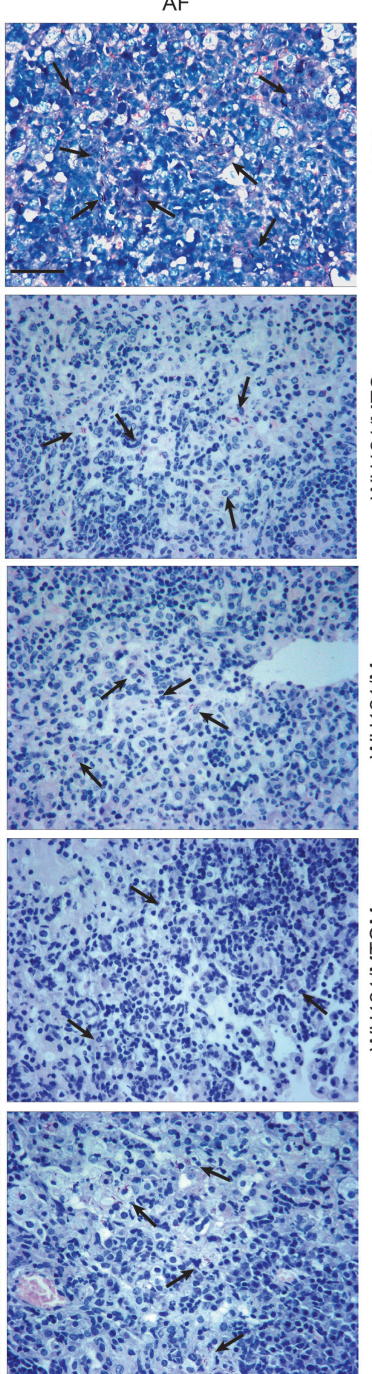
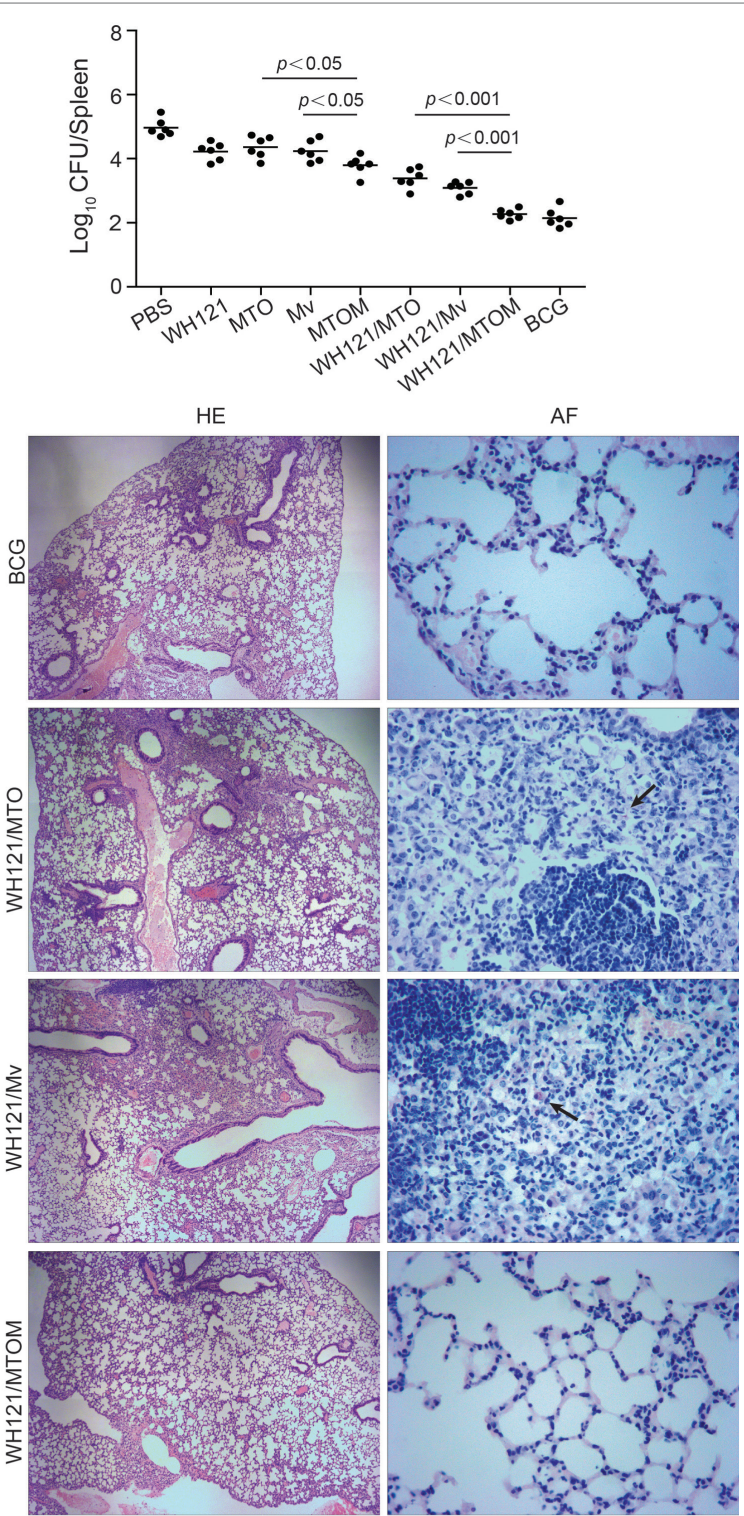

FIGURE 1 Comparison of protective efficacy of different vaccines. (A) Six weeks after final immunizations, C57BL/6 mice $(n=6)$ were intranasally challenged with about $100 \mathrm{CFU}$ virulent $M$. tb H37Rv strain. Four weeks after challenge, the bacterial load within lungs and spleens of mice in different groups was assessed and represented as mean ( \pm SEM) $\log _{10}$ CFU/organ $(n=6)$. (B) Lung tissue was sectioned and stained with hematoxylin and eosin (HE) (scale bar, $400 \mu$ m) and acid-fast (AF) staining (scale bar, $50 \mu \mathrm{m})$. Arrows indicate AF-positive bacteria.

splenocytes in the PBS control group secreted very low levels of IFN- $\gamma$, TNF- $\alpha$, and IL-2 in response to PPD or WH121 (Figure 3A). The levels of all antigen-specific Th1 cytokines except PPD-specific IFN- $\gamma$ were significantly higher in the MTOM alone group than in the PBS control group (Figure 3A). The WH121/MTOM vaccine induced higher levels of cytokine responses to PPD and WH121 than MTOM did $(P<0.05, P<0.001$, Figure 3A). Among all groups, the highest levels of PPD-specific Th1 cytokines were produced in the BCG group. However, mice vaccinated with WH121/ MTOM produced higher levels of WH121-specific TNF- $\alpha$ and IL-2 than BCG did $(P<0.001$, Figure 3A). In addition, MTOM adjuvant also stimulated higher levels of PPD-specific IFN- $\gamma$ and 

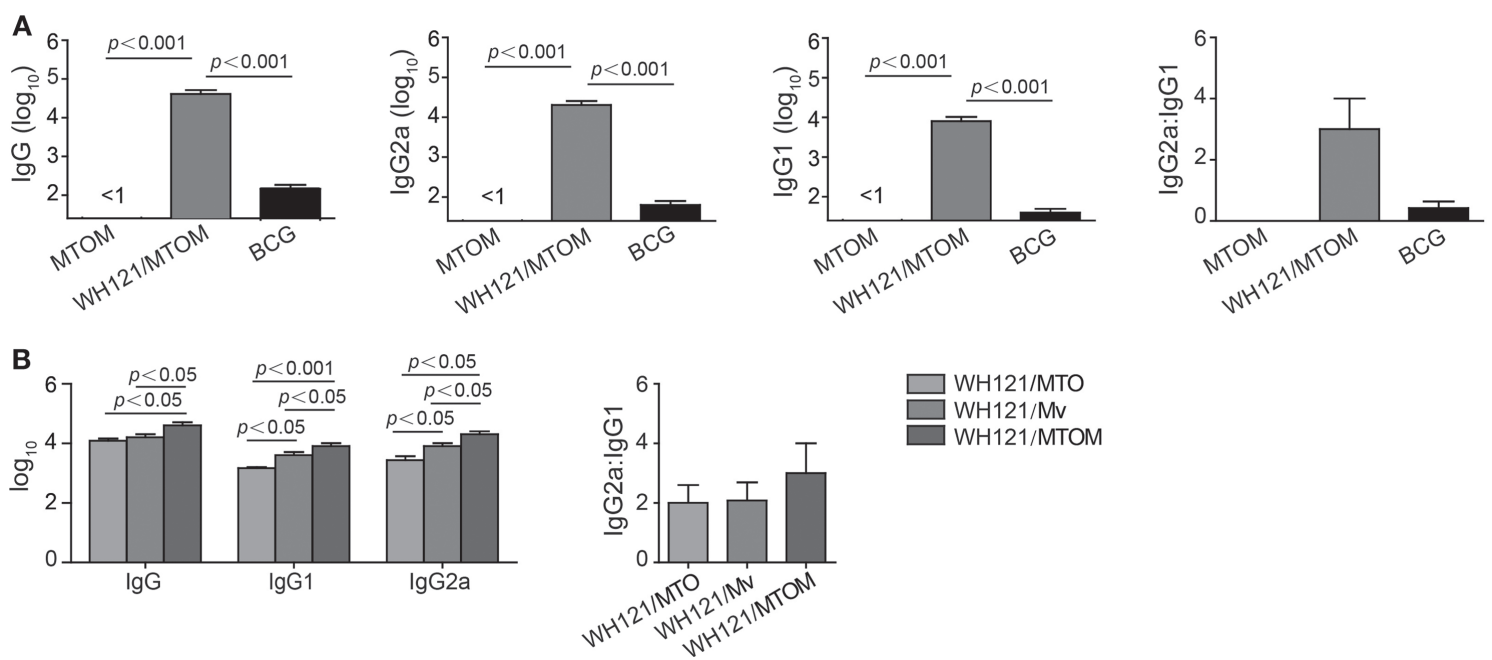

WH121/MTO

WH121/Mv

WH121/MTOM

FIGURE 2 | Effect of MTOM-adjuvanted WH121 protein on serum antibody levels in immunized mice. Six weeks after final immunization, the levels of serum IgG, IgG1, and IgG2c (replaced with IgG2a) antibodies from immunized mice were detected using ELISA. (A) WH121/MTOM induced IgG, IgG1, and IgG2a antibodies specific to WH121. (B) Comparison of antibody levels induced by WH121/MTO, WH121/Mv, and WH121/MTOM. Results are shown as mean ( \pm SEM) $\log _{10}$ end point titers and the ratio of IgG2a:lgG1 in the differently vaccinated groups $(n=3)$.

A
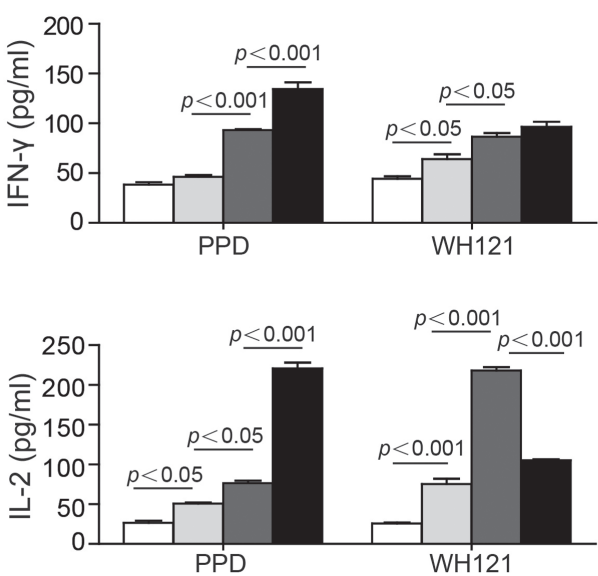

B

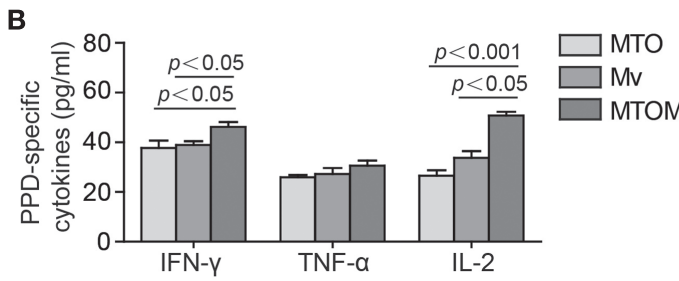

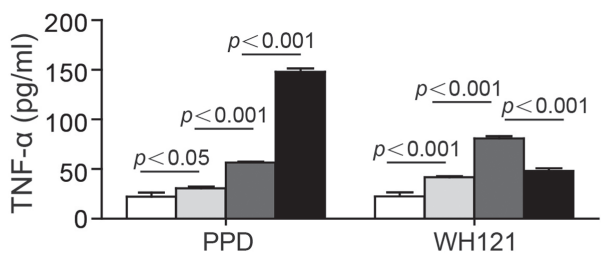
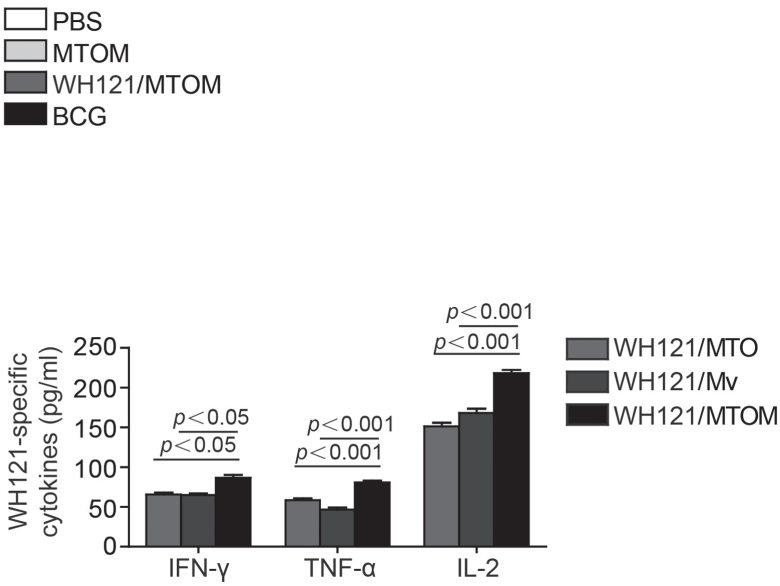

FIGURE 3 | The levels of IFN- $\boldsymbol{\gamma}$, TNF- $\boldsymbol{\alpha}$, and IL-2 secreted by splenocytes from different groups. Six weeks after the last immunization, splenocytes were collected from each mouse $(n=3)$. A total of $5 \times 10^{6}$ cells were added to each well of 24 -well microtiter plates and incubated with WH121 protein (10 $\mu$ g), PPD $\left(10 \mathrm{\mu g}\right.$ ), or complete RPMl1640 medium for $24 \mathrm{~h}$ (for IL-2 detection) or $72 \mathrm{~h}$ (for IFN- $\gamma$ and TNF- $\alpha$ detection) at $37^{\circ} \mathrm{C}$ in $5 \% \mathrm{CO}_{2}$. The cytokine concentrations in the suspension were determined by ELISA. (A) PPD- or WH121-specific cytokines induced by Bacillus Calmette-Guérin (BCG), PBS, WHI121/MTOM, and MTOM.

(B) PPD-specific cytokines induced by MTO, Mycobacterium vaccae (Mv) and MTOM, and WH121-specific cytokines induced by WH121/MTO, WH121/Mv, and WH121/MTOM. The results are shown as mean $\pm \mathrm{SD}(\mathrm{pg} / \mathrm{mL})$.

IL-2 than did MTO or Mv $(P<0.05, P<0.001$, Figure 3B). The levels of WH121-specific cytokines induced by WH121/MTOM were higher than those induced by WH121/MTO or WH121/ $\mathrm{Mv}(P<0.05, P<0.001$, Figure 3B). These results demonstrated that the adjuvant MTOM could promote the secretion of Th1 cytokines to a remarkable extent. The protection induced by MTOM formulated in the subunit vaccine was in a large part dependent on Th1-type responses. 


\section{Different Adjuvants Induced Different Multifunctional T Cell Subsets}

To assess the effects of WH121 protein formulated with different adjuvants on multifunctional $T$ cell subsets, biomarkers from splenocytes of vaccinated mice were stained and analyzed by multiparameter flow cytometry. The absolute number of PPD-specific single IFN $-\gamma^{+}$, single IL- $2^{+}$, single TNF- $\alpha^{+}$, IFN- $\gamma^{+}$TNF- $\alpha^{+}$, and IL- $2^{+}$TNF- $\alpha^{+}$CD $4^{+} \mathrm{T}$ cells was significantly higher in the BCG group than in the MTOM alone group $(P<0.05$, Figure 4A). All the combinations of $\mathrm{CD}^{+} \mathrm{T}$ cell cytokine responses to WH121 were elevated to a greater degree in the WH121/MTOM group than in the MTOM alone group $(P<0.05$, Figure $4 \mathrm{~A})$. In particular, WH121/MTOM showed higher levels of both PPD- and WH121-specific IFN- $\gamma^{+} \mathrm{IL}-2^{+} \mathrm{CD} 4^{+} \mathrm{T}$ cells than BCG $(P<0.05$, Figure $4 \mathrm{~A}$ ). In addition, relative to MTOM, the BCG vaccine induced mostly PPD-specific single IFN- $\gamma^{+}$and IFN- $\gamma^{+}$TNF- $\alpha^{+}$ $\mathrm{CD} 8^{+} \mathrm{T}$ cells, and the following cells: single IL- $2^{+}$, single TNF- $\alpha^{+}$, and IFN $-\gamma^{+}$TNF- $\alpha^{+}$IL- $2^{+}$CD $8^{+}$T cells; while the WH121/MTOM vaccine increased six combinations of $\mathrm{CD}^{+} \mathrm{T}$ cell responses to WH121 in addition to IFN- $\gamma^{+}$TNF- $\alpha^{+} \mathrm{CD}^{+} \mathrm{T}$ cells $(P<0.05$, Figure 4A). Notably, the frequency with which WH121-specific $\mathrm{CD}^{+} \mathrm{T}$ cells expressed single, double, or triple IL- $2^{+}$was higher in the WH121/MTOM group than in the BCG group $(P<0.05)$.

As shown in Figure 4B, MTO elicited mainly single IFN- $\gamma^{+}$ and single TNF- $\alpha^{+} \mathrm{CD} 4^{+}$and $\mathrm{CD} 8^{+} \mathrm{T}$ cells, which is consistent with previous findings (18). Mv induced mainly single IL-2 ${ }^{+}$ $\mathrm{CD}^{+} \mathrm{T}$ cells and single IFN- $\gamma^{+} \mathrm{CD} 8^{+} \mathrm{T}$ cells. Interestingly, MTOM elicited significantly more single, double, and triple IL- $2^{+}$, $\mathrm{CD} 4^{+}$, and CD $8^{+} \mathrm{T}$ cells than MTO or Mv $(P<0.05$, Figure 4B $)$. The multifunctional $\mathrm{T}$ cell responses induced by three WH121 subunit vaccines were essentially the same as their corresponding adjuvant alone. WH121/MTOM-vaccinated mice showed greater proportions of cells expressing IL-2 (either alone or co-expressed with other cytokines) than those vaccinated with WH121/MTO or WH121/Mv (Figure 4B).

\section{DISCUSSION}

Adjuvant plays a pivotal role in the development of new TB subunit vaccine candidates. We combined heat-killed $\mathrm{Mv}$ with MTO to develop a next-generation adjuvant, MTOM. Combined with a fusion protein WH121, it helped to evoke more robust antigen-specific Th1-type immune responses and generate more IL-2-positive multifunctional T cells than did MTO or Mv, thus providing the same protective efficacy against TB as the BCG vaccine. These indicate that the adjuvant MTOM is an efficient Th1-type immunostimulator and can produce TB vaccines with significantly improved immune effects.

$M . t b$ is infected naturally through respiratory route and engulfed by alveolar macrophages and dendritic cells (DCs). Pattern recognition receptors (PRRs) on the cell surface of APCs, including toll-like receptors (TLRs) and C-type lectin Mincle receptor, can recognize $M$. $t b$ and its components, which will lead to the activation of innate immunity and the initiation of adaptive immunity against $M$. $t b$ infection (22). We assumed that an effective subunit vaccine for TB might be developed based on the combination of different agonists of PRRs and multistage antigens of $M$. $t b$. The MF59 adjuvant is reported to target DCs, monocytes/macrophages, and granulocytes and to promote antigen presentation, immune cell differentiation, and the release of cytokines (23). MPLA is the first well-defined TLR4 agonist to induce robust $\mathrm{CD} 4^{+} \mathrm{T}$ cell responses and the production of type I interferons through TRIF and MyD88 signaling pathways $(24,25)$. The immunostimulation of TDB as the agonist of Mincle triggers the FcR $\gamma$-Syk-Card9 pathway in APCs, and then facilitates the activation of APCs and T cells (26). Fochesato et al. reported that MPLA is a major component of AS01 adjuvant, which has been used with varicella-zoster virus vaccine to increase the number of $\mathrm{CD}^{+} \mathrm{T}$ cells expressing IFN- $\gamma(27)$. Derrick et al. found the combinations of TDB and DDA boosted BCG to elevate the frequencies of $\mathrm{CD}^{+}$and $\mathrm{CD}^{+} \mathrm{T}$ cells expressing IFN- $\gamma$ and IFN- $\gamma /$ TNF- $\alpha$ (28). As expected, both MTO and MTOM containing MF59, MPLA, and TDB were able to increase the levels of IgG2a- and Th1-dominant cytokines and increase the number of multifunctional $\mathrm{CD} 4^{+}$and $\mathrm{CD} 8^{+} \mathrm{T}$ cells.

$M v$ is a non-pathogenic mycobacterium, which is the only immunoregulatory agent for the treatment of TB recommended by WHO (29). It is characterized by significant safety and efficacy (30). Unlike the materials used to produce the BCG vaccine, Mv is used as a heat-killed organism, so it provides less protection, and it may be more effective as an immunotherapy agent than as a vaccine agent. Mv has immunoregulatory components such as oligonucleotides (ODN) containing unmethylated $\mathrm{CpG}$ motifs that can be recognized by TLR9 and so enhance a Th1-biased immune response and $\mathrm{CD}^{+} \mathrm{T}$ cell-mediated response (31, 32). Mv might express some of the same antigens as $M$. $t b$ (33), which together are processed and cross-presented on MHC class I molecules on the APC, and so lead to the production of specific $\mathrm{CD} 8^{+}$cytotoxic $\mathrm{T}$ cells. Recently, in vitro and in vivo studies have also confirmed that $\mathrm{Mv}$ can downregulate Th2 and enhance Th1 responses through the induction of regulatory $\mathrm{T}$ cells and DCs (34).

In this study, we used multi-parameter flow cytometry to assess the magnitude and quality of the $\mathrm{T}$ cell responses in immunized mice. We found that $\mathrm{Mv}$ could increase the number of IL-2 ${ }^{+}$ $\mathrm{T}$ cells to a certain extent, and adding it into MTO triggered multifunctional $\mathrm{T}$ cell responses quite different from those induced by MTO or Mv alone. Correspondingly, the antigen-specific $\mathrm{CD}^{+}$and $\mathrm{CD} 8^{+} \mathrm{T}$ cells induced by WH121/MTO, WH121/Mv, and WH121/MTOM also presented phenotypic and functional heterogeneity. According to these results, the levels of single, double, and triple IL- $2^{+}, \mathrm{CD}^{+}$, and $\mathrm{CD} 8^{+} \mathrm{T}$ cells from MTOMimmunized mice were significantly higher than in the MTO and Mv groups. Likewise, mice vaccinated with WH121/MTOM showed many more T cells expressing IL-2 than WH121/MTOor WH121/Mv-treated mice. Although it is unclear whether the increase in IL-2 synthesis is correlated with greater vaccine effectiveness, elevated levels of IL-2 have been shown to contribute to the maintenance of cellular immunity and granuloma formation (35) and to augment effective immune responses via expansion of activated $\mathrm{CD}^{+} \mathrm{T}$ cells. They can also induce the expression of cytokines (e.g., IFN- $\gamma$ ) and the generation of immune memory $\mathrm{CD}^{+} \mathrm{T}$ cells $(36,37)$. The expression of IL-2 has been found to be associated with the successful long-term survival of activated 

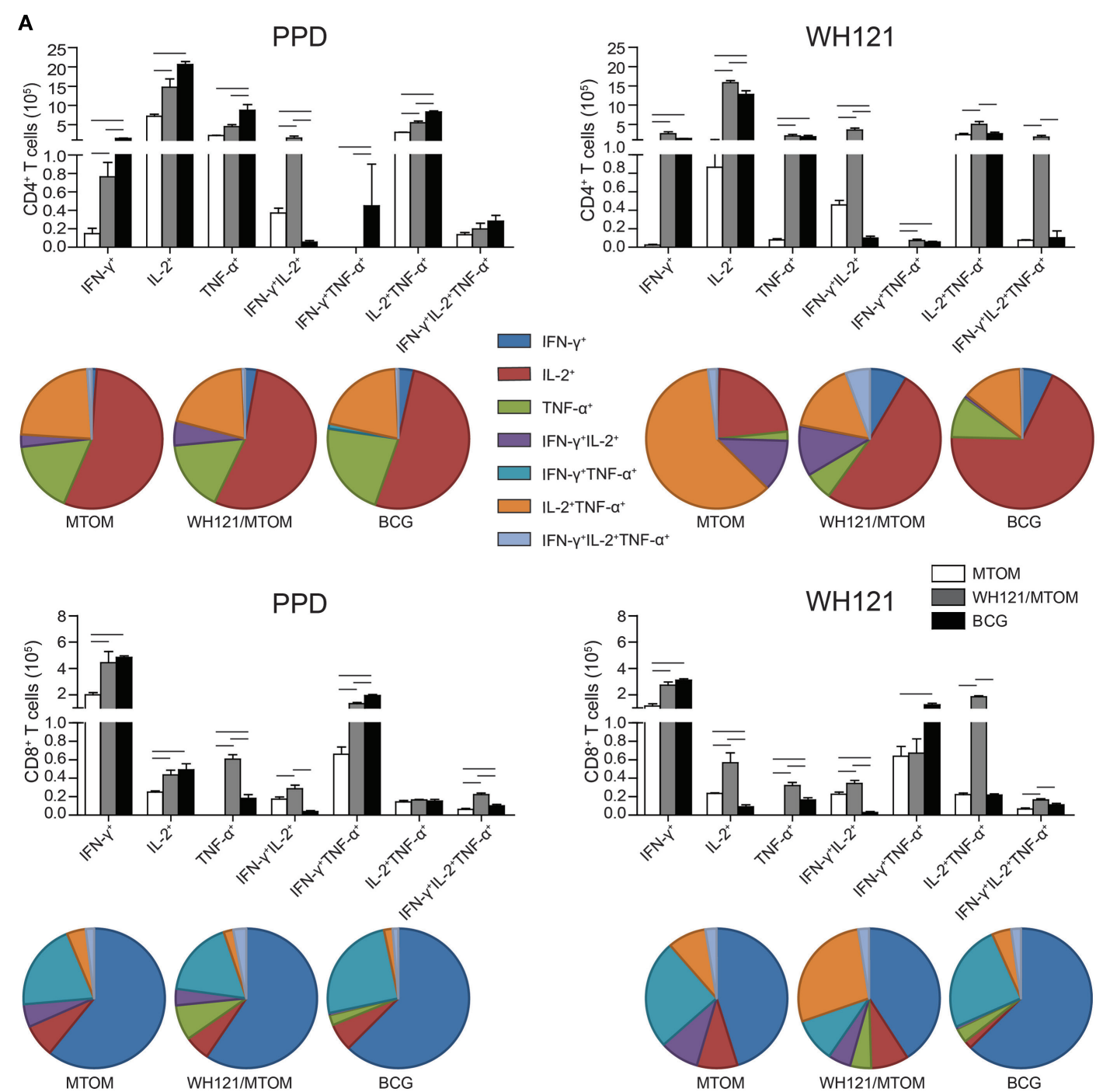

B
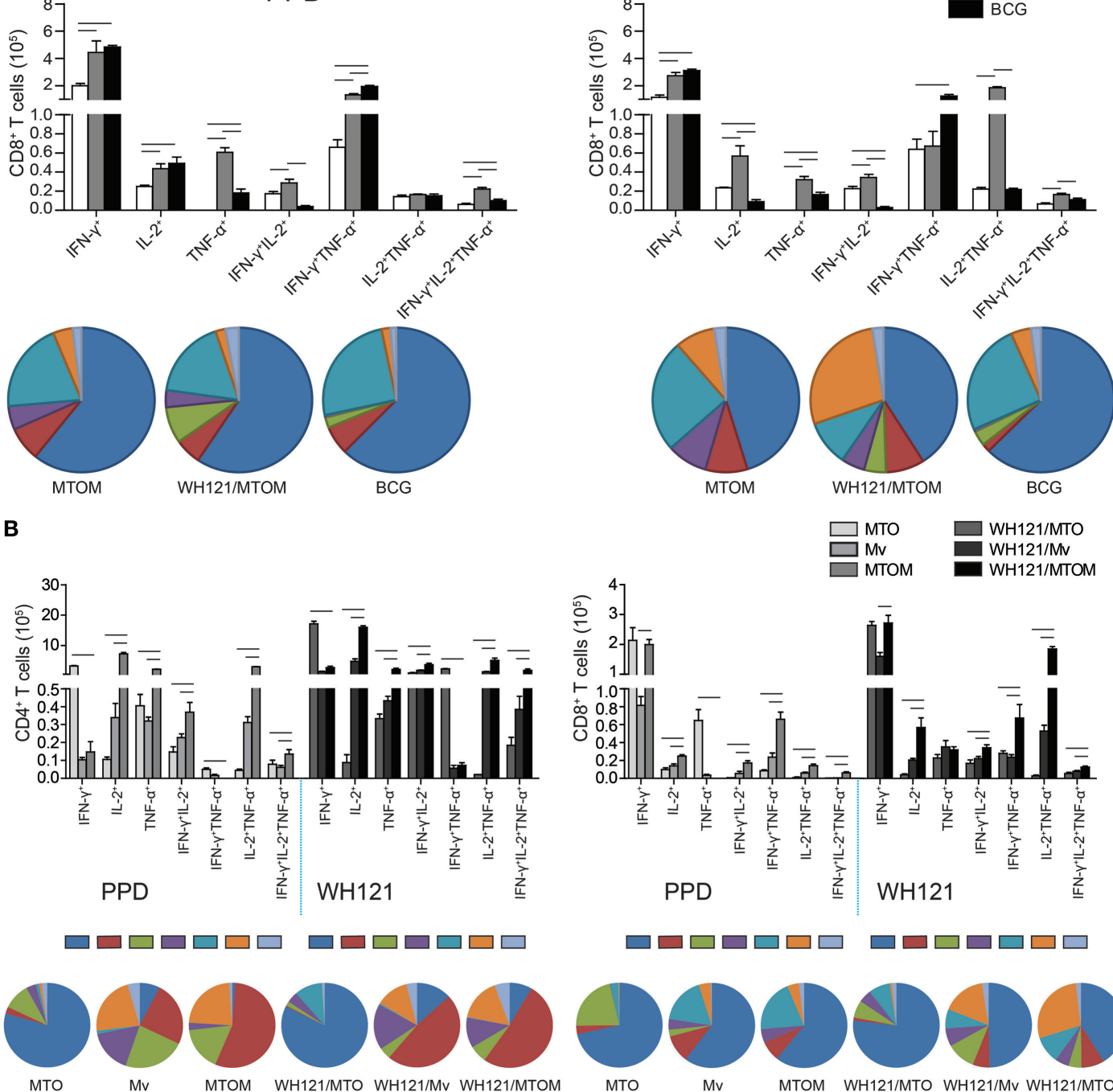

FIGURE $4 \mid$ Continued 
FIGURE 4 | Continued

PPD- and WH121-specific multifunctional CD4 ${ }^{+}$and CD8 $^{+} \mathbf{T}$ cell responses. Splenocytes from each mouse in each group were obtained and counted. A total of $5 \times 10^{6}$ cells per well were added to 24 -well plates and stimulated with WH121 protein (10 $\mu$ g) or PPD (10 $\mu$ g). Intracellular cytokine profiles for IFN- $\gamma$, TNF- $\alpha$, and IL-2 in individual cells were detected using multicolor flow cytometry by gating for CD4+ or CD8 ${ }^{+}$T cells. (A) The numbers of multifunctional CD4+ and CD8 ${ }^{+}$ T cells induced by WH121/MTOM. (B) PPD-specific CD4+ and CD8 ${ }^{+}$T cells elicited by MTO, Mycobacterium vaccae (Mv), and MTOM and WH121-specific CD4+ and CD8 ${ }^{+}$T cells induced by WH121/MTO, WH121/Mv, and WH121/MTOM. The number of cells in the PBS group, considered a background value, was subtracted. Each possible combination of cytokines is shown on the $x$-axis of the bar chart, and the absolute number of antigen-specific $T$ cells expressing any combination of cytokines is shown as mean \pm SEM in the different groups $(n=3)$. - represents $P<0.05$.

$\mathrm{CD}^{+}{ }^{+} \mathrm{T}$ cells in vivo (38). WH121/MTOM vaccine significantly increased the number of $\mathrm{CD}^{+}$or $\mathrm{CD}^{+} \mathrm{T}$ cells co-expressing multiple cytokines. Recent reports from various pathogens in different animal models have shown that multifunctional $\mathrm{T}$ cells that express IFN- $\gamma^{+}, \mathrm{TNF}-\alpha^{+}$, and IL- $2^{+}$are functionally superior to their single positive counterparts $(39,40)$. The concomitant production of the high levels of cytokines contributes to successful elimination of intracellular pathogens $(39,41)$. Aagaard et al. demonstrated that the H56/CAF01 vaccine improved the multifunctional $\mathrm{CD} 4^{+} \mathrm{T}$ cell populations secreting IFN- $\gamma^{+} \mathrm{IL}-2^{+}$ and IFN- $\gamma^{+}$IL- $2^{+}$TNF- $\alpha^{+}$and evoked a level of protection against TB comparable to that evoked by BCG (42). In our previous study (21), WH121/DMT elicited the higher numbers of IFN- $\gamma^{+}$IL- $2^{+}$ $\mathrm{CD}^{+} \mathrm{T}$ cells than did BCG. The same investigation of WH121 in the adjuvant of MTOM-vaccinated mice also showed pronounced induction of WH121-specific IFN- $\gamma^{+} \mathrm{IL}_{-} 2^{+}$and triple-positive $\mathrm{CD}^{+}{ }^{+} \mathrm{T}$ cells. Collectively, $\mathrm{Mv}$ improved the immunomodulatory effects of adjuvant MTO. The enhancement of anti-TB protection induced by WH121/MTOM is probably partially attributable to changes in multifunctional $\mathrm{T}$ cell responses. Specifically, the MTOM adjuvant promotes the generation and maintenance of Th1-type responses and IL-2 ${ }^{+} \mathrm{T}$ cells.

Because each component of the fusion protein, WH121, was not reported to function as adjuvant (10), WH121 protein-alonetreated mice only showed slight protection against $M$. $t b$ infection. Differential immunogenicity between different adjuvants alone, or between different adjuvanted WH121 vaccines, was analyzed in this study as previously performed $(43,44)$. All of these confirm that the synergistic action of both adjuvant and protein plays important roles in the efficacy of TB subunit vaccines. Differing from other studies $(43,44)$, BCG provided two to three log protections compared to PBS control in our experiments. Multiple factors such as different BCG substrain itself, immunization period, and infection time, dose and route might influence the results, as described earlier $(18,21)$. Moreover, the virulence of $M$. $t b \mathrm{H} 37 \mathrm{Rv}$ strain is highly variable and laboratory dependent (43, 44). In the mice immunized with WH121/MTOM, Th1 cytokine response was better compared to that induced by BCG immunization. However, the protective efficacy of WH121/MTOM was not significantly higher than BCG. Although Th1 responses are believed to be the most important protective mechanism against $M$. $t b$, the role of innate immunity also cannot be ignored (45). As a live bacterium, the protective mechanism of BCG has remarkable difference from that of subunit vaccine. As a result, more research is needed to further enhance the immunogenicity of our subunit protein vaccine, and to optimize dose administration.

In conclusion, this is the first report to combine Mv to MTO to form a new water-in-oil adjuvant, called MTOM. This adjuvant is found to augment Th1-type responses and IL-2 ${ }^{+} \mathrm{T}$ cells, thus could be used for the development of vaccine candidates against other intracellular pathogens. Our studies also demonstrate that MTOM-based WH121 vaccine has better immunogenicity and protection than other adjuvanted vaccines and is comparable to BCG in effectiveness. This work may help to accelerate the development of TB vaccines, and it lays the foundation for further preclinical evaluation of WH121/MTOM in other animal models.

\section{MATERIALS AND METHODS}

\section{Preparation of Adjuvants and Vaccines}

The WH121 fusion protein expressed by a genetically engineered expression system in $E$. coli was constructed and prepared in our laboratory. Water-in-oil adjuvant, MTO, was prepared as described previously (18). Mv was cultivated in sterile medium ( $2.5 \mathrm{~g} / \mathrm{L}$ yeast extract, $5 \mathrm{~g} / \mathrm{L}$ tryptone, $1 \mathrm{~g} / \mathrm{L}$ glucose) at $37^{\circ} \mathrm{C}$. The mycobacteria were harvested by centrifugation and transferred to sterile 7H9 medium (Difco, USA, Cat. no. 0189627) with $10 \%$ $\mathrm{ADC}$ and $0.5 \%$ glycerol at $37^{\circ} \mathrm{C}$ for $24 \mathrm{~h}$. Culture supernatant was discarded after centrifugation. Then, the precipitate was concentrated and weighed. Heat-killed Mv was prepared from the mycobacterial pellet resuspended in $\mathrm{PBS}$ at $10 \mathrm{mg} / \mathrm{mL}$ (equivalent to $10^{10} \mathrm{CFU} / \mathrm{mL}$ ) and autoclaved for $15 \mathrm{~min}$ at $121^{\circ} \mathrm{C}$. The lysate of $\mathrm{Mv}$ was diluted to $5 \mathrm{mg} / \mathrm{mL}$ (19). MTOM adjuvant was prepared as an evenly emulsified mixture of $1 \mathrm{~mL}$ MTO and $1 \mathrm{~mL}$ Mv lysate solution. Finally, $200 \mu \mathrm{L}$ of immunization dose of each WH121 subunit vaccines was prepared by mixing $20 \mu \mathrm{g}$ WH121 protein and $50 \mu \mathrm{L}$ MTO or Mv or $100 \mu \mathrm{L}$ MTOM in $10 \mathrm{mM}$ Tris buffer, respectively.

\section{Mice and Immunizations}

C57BL/ 6 female mice aged 6-8 weeks were obtained from the Center for Animal Experiments of Wuhan University (Wuhan, China) and maintained under specific pathogen-free conditions in a BSL-3 laboratory. For this study, $200 \mu \mathrm{L}$ of WH121 vaccine preparations was given to mice subcutaneously (s.c.) twice at 3-week intervals. WH121 protein and adjuvants, MTO, Mv, and MTOM, were similarly administered for comparison. The BCG China vaccine was used as a positive control, which was administered once $\left(1 \times 10^{6} \mathrm{CFU}\right)$ s.c. at the time of the first vaccination. PBS served as a negative control.

\section{Infection with $M$. tb H37Rv and Evaluation of Vaccine-induced Immune Protection}

M. $t b \mathrm{H} 37 \mathrm{Rv}$ was grown to mid-log phase in sterile Middlebrook 7 H11 agar (Difco, USA, Cat. no. 4364531) with 10\% ADC and 
$0.5 \%$ glycerol, and harvested and weighed. Then, the bacterial suspension was prepared in $\mathrm{PBS}$ at $0.1 \mathrm{mg} / \mathrm{mL}$ (about equivalent to $10^{6} \mathrm{CFU} / \mathrm{mL}$ ) by a sterile glass grinder. Six weeks after their last immunization, mice in each group were first anesthetized by intraperitoneal injection with $120 \mu \mathrm{L}$ of $0.8 \%$ pentobarbital sodium, namely $0.96 \mathrm{mg} / 15 \mathrm{~g}$ weight of mice. $20 \mu \mathrm{L}$ of the bacterial suspension in a microinjector was then used to infect each mouse intranasally $(18,21,46,47)$. After infection, three mice in the PBS control group were killed on the next day, and the actual infection dose was determined by enumeration of lung bacterial load. Four weeks later, the mice were sacrificed for the evaluation of protective efficacy. Lungs and spleens were removed aseptically and homogenized in sterile 0.05\% PBSTween 80 , then plated at 10-fold serial dilutions on Middlebrook 7H11 agar plates. 2-thiophenecarboxylic acid hydrazide $(2 \mu \mathrm{g} /$ $\mathrm{mL}$ ) was supplemented to plates to inhibit the possible growth of residual BCG in the BCG group. Plates were cultured at $37^{\circ} \mathrm{C}$ for 4 weeks, and the bacterial load was shown as mean $\left(\log _{10}\right.$ $\mathrm{CFU}) \pm$ SEM per organ for each group $(n=6)$. Right lung lobes from the differently vaccinated groups $(n=3)$ were fixed in $10 \%$ buffered formaldehyde and embedded in paraffin. Slices ( $5 \mu \mathrm{m}$ thick) were cut and stained with hematoxylin and eosin (HE) or AF staining. Pathological changes were analyzed with light microscopy (Nikon ECLIPSE E100, Japan).

\section{Detection of WH121-Specific Antibody Levels}

Six weeks after the last immunization, sera were collected from vaccinated mice. WH121-specific end point titers for IgG1, IgG2c, and total IgG antibodies were detected by ELISA. Using previously described methods (48), microtiter plates were pre-coated with $100 \mu \mathrm{L}$ WH121 protein $(5 \mu \mathrm{g} / \mathrm{mL})$ in carbonate/bicarbonate buffer ( $\mathrm{pH}$ 9.6) overnight at $4^{\circ} \mathrm{C}$ and blocked with $1 \%$ BSA in PBST for $1 \mathrm{~h}$ at $37^{\circ} \mathrm{C}$. Horseradish peroxidase-conjugated rabbit anti-mouse IgG (1/5,000; Abcam, UK, Cat. no. Ab6789), IgG1 (1/10,000; Abcam, UK, Cat. no. Ab97240), or IgG2c (1/10,000; Abcam, UK, Cat. no. Ab97255) were added to the plates. $100 \mu \mathrm{L}$ $3,3^{\prime} 5,5^{\prime}$-tetramethylbenzidine substrate was used for visualization at $37^{\circ} \mathrm{C}$. Plates were read at $450 \mathrm{~nm}$ using a Multiskan ELISA reader (Bio-tek, USA). Antibody titers in each group were determined by comparison to the PBS control group. The results are expressed as mean $( \pm$ SEM $) \log _{10}$ end point titers, and as the ratio of IgG2c/IgG1.

\section{Antigen-Specific IFN- $\gamma$, IL-2, and TNF- $\alpha$ ELISA}

Spleens were removed aseptically from immunized mice. Splenocytes were counted and plated at $5 \times 10^{6}$ in each well of 24-well microtiter plate for $24 \mathrm{~h}$ (for IL-2 determination) and $72 \mathrm{~h}$ (for IFN- $\gamma$ and TNF- $\alpha$ detection) at $37^{\circ} \mathrm{C}$ with $\mathrm{WH} 121$ $(10 \mu \mathrm{g} / \mathrm{mL})$. RPMI1640 medium served as a negative control; PPD (10 $\mu \mathrm{g} / \mathrm{mL}$; Statens Serum Institut, Denmark) served as a positive control. Supernatants were collected from three separate wells to assess the levels of IFN- $\gamma$, IL-2, and TNF- $\alpha$ using mouse IFN- $\gamma$ (EK2802), IL-2(EK2025), and TNF- $\alpha$ ELISA kits (EK2822, all from Multi Sciences Ltd., Hangzhou, China) according to the manufacturer's instructions. The results in this study are expressed as mean $\pm \mathrm{SD}(\mathrm{pg} / \mathrm{mL})$ of each group $(n=3)$.

\section{Detection of Multifunctional T Cells with Flow Cytometry}

Splenocytes $\left(5 \times 10^{6}\right)$ from three individual mice per group were prepared as mentioned earlier and plated in a 24 -well plate, stimulated with WH121 $(10 \mu \mathrm{g} / \mathrm{mL})$ for $16 \mathrm{~h}$, and incubated for another $4-6 \mathrm{~h}$ after the addition of $3 \mu \mathrm{g} / \mathrm{mL}$ brefeldin A and $2 \mu \mathrm{M}$ monensin solution (eBioscience, USA). RPMI1640 medium provided a negative control, and PPD $(10 \mu \mathrm{g} / \mathrm{mL})$ was used as positive control. A cell stimulation cocktail $(1 \mu \mathrm{g} / \mathrm{mL}$; eBioscience, USA) was used to monitor cell responses. After washing in FACS buffer (1\% FCS-PBS), the surface markers of cells were stained using anti-CD3-FITC (eBioscience, USA, Cat. no. 11-0032), anti-CD4-APC-cy7 (BD Biosciences, USA, Cat. no. BD-557871), and anti-CD8a PE (eBioscience, USA, Cat. no. 12-0081) $\mathrm{mAbs}$ at $4^{\circ} \mathrm{C}$ for $30 \mathrm{~min}$ in the dark. Cells were washed again and permeabilized using an Intracellular Fixation and Permeabilization Buffer Kit (eBioscience, USA). AntiIFN- $\gamma$ PerCP-Cyanine5.5 (eBioscience, USA, Cat. no. 45-7311), anti-TNF-PE-Cy ${ }^{\mathrm{TM}} 7$ (eBioscience, USA, Cat. no. 25-7321), and anti-IL-2 APC (eBioscience, USA, Cat. no. 17-7021) mAbs were used for intracellular staining. The absolute number of $\mathrm{CD}^{+}$and $\mathrm{CD}^{+} \mathrm{T}$ cell subsets secreting single, double, or triple cytokines was determined using the LSRII multicolor flow cytometer (BD Biosciences, USA) and analyzed using FlowJo software. To provide baseline normal values, the number of cells from three mice in the PBS group was determined. The results are shown as mean \pm SEM per group $(n=3)$.

\section{Statistical Analysis}

Graph Pad Prism 5 software was used to analyze the data. Statistical analysis was performed using SPSS 18.0 software. Oneway ANOVA or $t$-test was used to assess the difference between the different groups. $P$-values $<0.05$ were considered significant.

\section{ETHICS STATEMENT}

The experiments described in this study were performed in accordance with the guidelines of the Chinese Council on Animal Care. The research protocols were approved by the Committee on the Ethics of Animal Experiments of Huazhong University of Science and Technology.

\section{AUTHOR CONTRIBUTIONS}

XF designed this study. XW performed preceding experiments and assisted in data collection. QY conducted the experiments, analyzed the data, and prepared the manuscript. XF and QY revised the manuscript.

\section{FUNDING}

This work was supported by the grants of the National High Technology Research and Development Program of China 
(863 program; No. 2012AA02A401), the National Megaprojects of Science Research for the 12th 5-year Plan of China (No. 2012ZX10003008-005), the Fundamental Research
Funds for the Central Universities (HUST: 2015MS098 and 2015ZZGH012), and Wuhan Bureau of Science and Technology (No. 2015060101010029).

\section{REFERENCES}

1. Global Tuberculosis Report 2016. Geneva, Switzerland: United Nations, World Health Organization (WHO) (2016). Available from: http://www.who.int/tb/ publications/global_report/en/

2. Rodrigues LC, Diwan VK, Wheeler JG. Protective effect of BCG against tuberculous meningitis and miliary tuberculosis: a meta-analysis. Int J Epidemiol (1993) 22(6):1154-8. doi:10.1093/ije/22.6.1154

3. Cooper AM. Cell-mediated immune responses in tuberculosis. Annu Rev Immunol (2009) 27:393-422. doi:10.1146/annurev.immunol.021908.132703

4. Sakai S, Mayer-Barber KD, Barber DL. Defining features of protective CD4 $\mathrm{T}$ cell responses to Mycobacterium tuberculosis. Curr Opin Immunol (2014) 29:137-42. doi:10.1016/j.coi.2014.06.003

5. Yao S, Huang D, Chen CY, Halliday L, Wang RC, Chen ZW. CD4+ T cells contain early extrapulmonary tuberculosis (TB) dissemination and rapid TB progression and sustain multieffector functions of CD8+ T and CD3lymphocytes: mechanisms of CD4+ T cell immunity. JImmunol (2014) 192(5):2120-32. doi:10.4049/jimmunol.1301373

6. Sud D, Bigbee C, Flynn JL, Kirschner DE. Contribution of CD8+ T cells to control of Mycobacterium tuberculosis infection. JImmunol (2006) 176(7):4296-314. doi:10.4049/jimmunol.176.7.4296

7. Jasenosky LD, Scriba TJ, Hanekom WA, Goldfeld AE. T cells and adaptive immunity to Mycobacterium tuberculosis in humans. Immunol Rev (2015) 264(1):74-87. doi:10.1111/imr.12274

8. Delany I, Rappuoli R, De Gregorio E. Vaccines for the 21st century. EMBO Mol Med (2014) 6(6):708-20. doi:10.1002/emmm.201403876

9. Kaufmann SH. Tuberculosis vaccine development at a divide. Curr Opin Pulm Med (2014) 20(3):294-300. doi:10.1097/MCP.0000000000000041

10. Moyle PM. Biotechnology approaches to produce potent, self-adjuvanting antigen-adjuvant fusion protein subunit vaccines. Biotechnol Adv (2017) 35(3):375-89. doi:10.1016/j.biotechadv.2017.03.005

11. Oleszycka E, Lavelle EC. Immunomodulatory properties of the vaccine adjuvant alum. Curr Opin Immunol (2014) 28:1-5. doi:10.1016/j.coi.2013.12.007

12. Knuf M, Leroux-Roels G, Rümke HC, Abarca K, Rivera L, Lattanzi M, et al. Safety and immunogenicity of an MF59-adjuvanted A/H1N1 pandemic influenza vaccine in children from three to seventeen years of age. Vaccine (2015) 33(1):174-81. doi:10.1016/j.vaccine.2014.10.085

13. Morel S, Didierlaurent A, Bourguignon P, Delhaye S, Baras B, Jacob V, et al. Adjuvant System AS03 containing alpha-tocopherol modulates innate immune response and leads to improved adaptive immunity. Vaccine (2011) 29(13):2461-73. doi:10.1016/j.vaccine.2011.01.011

14. Garcon N, Wettendorff M, Van Mechelen M. Role of AS04 in human papillomavirus vaccine: mode of action and clinical profile. Expert Opin Biol Ther (2011) 11(5):667-77. doi:10.1517/14712598.2011.573624

15. Leroux-Roels G. Old and new adjuvants for hepatitis B vaccines. Med Microbiol Immunol (2015) 204(1):69-78. doi:10.1007/s00430-014-0375-9

16. Agger EM. Novel adjuvant formulations for delivery of anti-tuberculosis vaccine candidates. Adv Drug Deliv Rev (2016) 102:73-82. doi:10.1016/j. addr.2015.11.012

17. Desel C, Werninghaus K, Ritter M, Jozefowski K, Wenzel J, Russkamp N, et al. The Mincle-activating adjuvant TDB induces MyD88-dependent Th1 and Th17 responses through IL-1R signaling. PLoS One (2013) 8(1):e53531. doi:10.1371/journal.pone.0053531

18. Wang X, Zhang J, Liang J, Zhang Y, Teng X, Yuan X, et al. Protection against Mycobacterium tuberculosis infection offered by a new multistage subunit vaccine correlates with increased number of IFN- $\gamma+$ IL-2+ CD4+ and IFN- $\gamma+$ CD8+ T cells. PLoS One (2015) 10(3):e0122560. doi:10.1371/journal. pone. 0122560

19. Skinner MA, Yuan S, Prestidge R, Chuk D, Watson JD, Tan PL. Immunization with heat-killed Mycobacterium vaccae stimulates CD8+ cytotoxic T cells specific for macrophages infected with Mycobacterium tuberculosis. Infect Immun (1997) 65(11):4525-30.

20. Liu L, Fu R, Yuan X, Shi C, Wang S, Lu X, et al. Differential immune responses and protective effects in avirulent mycobacterial strains vaccinated BALB/c mice. Curr Microbiol (2015) 71(1):129-35. doi:10.1007/s00284-0150837-3

21. Ma J, Tian M, Fan X, Yu Q, Jing Y, Wang W, et al. Mycobacterium tuberculosis multistage antigens confer comprehensive protection against pre- and post-exposure infections by driving Th1-type T cell immunity. Oncotarget (2016) 7(39):63804-15. doi:10.18632/oncotarget.11542

22. Shaler CR, Horvath C, Lai R, Xing Z. Understanding delayed T-cell priming, lung recruitment, and airway luminal T-cell responses in host defense against pulmonary tuberculosis. Clin Dev Immunol (2012) 2012:628293. doi:10.1155/2012/628293

23. Mulligan MJ, Bernstein DI, Frey S, Winokur P, Rouphael N, Dickey M, et al. Point-of-use mixing of influenza H5N1 vaccine and MF59 adjuvant for pandemic vaccination preparedness: antibody responses and safety. A phase 1 clinical trial. Open Forum Infect Dis (2014) 1(3):ofu102. doi:10.1093/ofid/ ofu102

24. Pouliot K, Buglione-Corbett R, Marty-Roix R, Montminy-Paquette S, West K, Wang S, et al. Contribution of TLR4 and MyD88 for adjuvant monophosphoryl lipid A (MPLA) activity in a DNA prime-protein boost HIV-1 vaccine. Vaccine (2014) 32(39):5049-56. doi:10.1016/j.vaccine.2014.07.010

25. Shen H, Tesar BM, Walker WE, Goldstein DR. Dual signaling of MyD88 and TRIF is critical for maximal TLR4-induced dendritic cell maturation. J Immunol (2008) 181(3):1849-58. doi:10.4049/jimmunol.181.3.1849

26. Werninghaus K, Babiak A, Gross O, Hölscher C, Dietrich H, Agger EM, et al. Adjuvanticity of a synthetic cord factor analogue for subunit Mycobacterium tuberculosis vaccination requires FcRgamma-Syk-Card9-dependent innate immune activation. JExp Med (2009) 206(1):89-97. doi:10.1084/jem. 20081445

27. Fochesato M, Dendouga N, Boxus M. Comparative preclinical evaluation of AS01 versus other adjuvant systems in a candidate herpes zoster glycoprotein E subunit vaccine. Hum Vaccin Immunother (2016) 12(8):2092-5. doi:10.108 0/21645515.2016.1154247

28. Derrick SC, Yang A, Parra M, Kolibab K, Morris SL. Effect of cationic liposomes on BCG trafficking and vaccine-induced immune responses following a subcutaneous immunization in mice. Vaccine (2015) 33(1):126-32. doi:10.1016/j.vaccine.2014.11.004

29. WHO/TB/91.162, Tuberculosis Research and Development. Geneva, Switzerland: United Nations, World Health Organization (WHO) (1991) Available from: http://www.who.int/tb/publications/1991/en/

30. Waddell RD, Chintu C, Lein AD, Zumla A, Karagas MR, Baboo KS, et al. Safety and immunogenicity of a five-dose series of inactivated Mycobacterium vaccae vaccination for the prevention of HIV-associated tuberculosis. Clin Infect Dis (2000) 30(Suppl 3):S309-15. doi:10.1086/313880

31. Zuany-Amorim C, Manlius C, Trifilieff A, Brunet LR, Rook G, Bowen G, et al. Long-term protective and antigen-specific effect of heat-killed Mycobacterium vaccae in a murine model of allergic pulmonary inflammation. J Immunol (2002) 169(3):1492-9. doi:10.4049/jimmunol.169.3.1492

32. Scheiermann J, Klinman DM. Clinical evaluation of CpG oligonucleotides as adjuvants for vaccines targeting infectious diseases and cancer. Vaccine (2014) 32(48):6377-89. doi:10.1016/j.vaccine.2014.06.065

33. Stanford J, Stanford C, Grange J. Immunotherapy with Mycobacterium vaccae in the treatment of tuberculosis. Front Biosci (2004) 9:1701-19. doi:10.2741/1292

34. Bazzi S, Modjtahedi H, Mudan S, Akle C, Bahr GM. Analysis of the immunomodulatory properties of two heat-killed mycobacterial preparations in a human whole blood model. Immunobiology (2015) 220(12):1293-304. doi:10.1016/j.imbio.2015.07.015

35. Yamamoto $\mathrm{T}$, Iwamoto $\mathrm{N}$, Yamamoto $\mathrm{H}$, Tsukamoto $\mathrm{T}$, Kuwano $\mathrm{T}$, Takeda A, et al. Polyfunctional CD4+ T-cell induction in neutralizing antibody-triggered control of simian immunodeficiency virus infection. J Virol (2009) 83(11):5514-24. doi:10.1128/JVI.00145-09 
36. Derrick SC, Yabe IM, Yang A, Morris SL. Vaccine-induced anti-tuberculosis protective immunity in mice correlates with the magnitude and quality of multifunctional CD4 T cells. Vaccine (2011) 29(16):2902-9. doi:10.1016/j. vaccine.2011.02.010

37. Oh HS, Choi BK, Kim YH, Lee DG, Hwang S, Lee MJ, et al. 4-1BB signaling enhances primary and secondary population expansion of CD8+ T cells by maximizing autocrine IL-2/IL-2 receptor signaling. PLoS One (2015) 10(5):e0126765. doi:10.1371/journal.pone.0126765

38. Dooms H, Kahn E, Knoechel B, Abbas AK. IL-2 induces a competitive survival advantage in T lymphocytes. J Immunol (2004) 172(10):5973-9. doi:10.4049/ jimmunol.172.10.5973

39. Darrah PA, Patel DT, De Luca PM, Lindsay RW, Davey DF, Flynn BJ, et al. Multifunctional TH1 cells define a correlate of vaccine-mediated protection against Leishmania major. Nat Med (2007) 13(7):843-50. doi:10.1038/nm1592

40. Kannanganat S, Ibegbu C, Chennareddi L, Robinson HL, Amara RR. Multiplecytokine-producing antiviral CD4 T cells are functionally superior to singlecytokine-producing cells. J Virol (2007) 81(16):8468-76. doi:10.1128/ JVI.00228-07

41. Seder RA, Darrah PA, Roederer M. T-cell quality in memory and protection: implications for vaccine design. Nat Rev Immunol (2008) 8(4):247-58. doi:10.1038/nri2274

42. Aagaard C, Hoang T, Dietrich J, Cardona PJ, Izzo A, Dolganov G, et al. A multistage tuberculosis vaccine that confers efficient protection before and after exposure. Nat Med (2011) 17(2):189-94. doi:10.1038/nm.2285

43. Baldwin SL, Bertholet S, Reese VA, Ching LK, Reed SG, Coler RN. The importance of adjuvant formulation in the development of a tuberculosis vaccine. J Immunol (2012) 188(5):2189-97. doi:10.4049/jimmunol.1102696

44. Bertholet S, Ireton GC, Ordway DJ, Windish HP, Pine SO, Kahn M, et al. A defined tuberculosis vaccine candidate boosts BCG and protects against multidrug-resistant Mycobacterium tuberculosis. Sci Transl Med (2010) 2(53):53ra74. doi:10.1126/scitranslmed.3001094

45. Philips JA, Ernst JD. Tuberculosis pathogenesis and immunity. Annu Rev Pathol (2012) 7:353-84. doi:10.1146/annurev-pathol-011811-132458

46. Damjanovic D, Khera A, Afkhami S, Lai R, Zganiacz A, Jeyanathan M, et al. Age at Mycobacterium bovis BCG priming has limited impact on anti-tuberculosis immunity boosted by respiratory mucosal AdHu5Ag85A immunization in a murine model. PLoS One (2015) 10(6):e0131175. doi:10.1371/journal. pone.0131175

47. Mu J, Jeyanathan M, Small CL, ZhangX, Roediger E, Feng X, et al. Immunization with a bivalent adenovirus-vectored tuberculosis vaccine provides markedly improved protection over its monovalent counterpart against pulmonary tuberculosis. Mol Ther (2009) 17(6):1093-100. doi:10.1038/mt.2009.60

48. Teng X, Tian M, Li J, Tan S, Yuan X, Yu Q, et al. Immunogenicity and protective efficacy of DMT liposome-adjuvanted tuberculosis subunit CTT3H vaccine. Hum Vaccin Immunother (2015) 11(6):1456-64. doi:10.1080/21645515.2015. 1037057

Conflict of Interest Statement: The authors declare that the research was conducted in the absence of any commercial or financial relationships that could be construed as a potential conflict of interest.

Copyright (C) 2017 Yu, Wang and Fan. This is an open-access article distributed under the terms of the Creative Commons Attribution License (CC BY). The use, distribution or reproduction in other forums is permitted, provided the original author $(s)$ or licensor are credited and that the original publication in this journal is cited, in accordance with accepted academic practice. No use, distribution or reproduction is permitted which does not comply with these terms. 